\title{
Non-intubated uniportal video-assisted thoracoscopy for sleeve lobectomy in a patient with severe chronic obstructive pulmonary disease: a case report
}

\author{
Lihua Dai ${ }^{1 \#}$, Zhigang Chen ${ }^{1 \#}$, Jacopo Vannucci $^{2,3}$, Jianqiao Cai $^{2}, \mathrm{Xin}_{\mathrm{Lv}^{1}}$, Jianming Liu ${ }^{1}$, Lei Jiang ${ }^{2}$ \\ ${ }^{1}$ Department of Anesthesiology, Shanghai Pulmonary Hospital, Tongji University, Shanghai, China; ${ }^{2}$ Department of Thoracic Surgery, Shanghai \\ Pulmonary Hospital, Tongji University, Shanghai, China; ${ }^{3}$ Department of Thoracic Surgery, University of Rome Sapienza, Policlinico Umberto, \\ Rome, Italy \\ \#These authors contributed equally to this work. \\ Correspondence to: Lei Jiang. Department of Thoracic Surgery, Shanghai Pulmonary Hospital, No. 507 Zhengmin Rd. Yangpu District, Shanghai \\ 200433, China. Email: jiangleiem@aliyun.com.
}

\begin{abstract}
Patients with compromised lung function undergoing uniportal video-assisted thoracoscopic surgeries (VATS) are at high risk for postoperative pulmonary complications. Ventilation technique for these patients should attempt to minimize the potential lung injury as well as to obtain a satisfactory surgical field and provide adequate intraoperative oxygenation. Non-intubated thoracic surgery has been reported to be associated with less ventilatory impairment compared to general anesthesia with double lumen tube intubation. However, this technique in complex surgeries such as sleeve lobectomy is not standardized yet. We described a 71-year-old male with severe chronic obstructive pulmonary disease (COPD) and primary carcinoma obstructing the right upper lobe. Uniportal VATS right upper sleeve lobectomy was performed under non-intubated anesthesia. A cross-field bronchial suction catheter was inserted through the open end of the right main bronchus to provide left lung oxygen delivery during the anastomosis maneuvers. Satisfactory operative conditions as well as adequate oxygenation was achieved using this technique. No signs of pulmonary complications or any other discomfort was observed during the patient's hospital stay. Thus, we suggest that non-intubated anesthesia could be a safe or even better option in the case of a patient with reduced lung function for complex uniportal VATS surgeries like sleeve lobectomy.
\end{abstract}

Keywords: Non-intubated; uniportal video-assisted thoracoscopic surgery; sleeve lobectomy; chronic obstructive pulmonary disease (COPD); case report

Received: 13 September 2020; Accepted: 30 December 2020; Published: 20 October 2021.

doi: $10.21037 /$ jovs-20-158

View this article at: http://dx.doi.org/10.21037/jovs-20-158

\section{Introduction}

General anesthesia with double lumen tube intubation to achieve one-lung ventilation is the standard of care for video-assisted thoracic surgeries (VATS) but this technique is associated with many possible postoperative complications, especially in patients with pulmonary dysfunction (1). Non-intubated anesthesia with spontaneous ventilation could be a valuable option in case of impaired respiratory condition as it shows the advantages of avoiding intubation and ventilator-related complications and lung injuries $(2,3)$. However, the ventilation technique remains challenging for the anesthesiologists when complex techniques such as sleeve lobectomy under non-intubated uniportal VATS is planned to be performed.

A careful respiratory management is essential as well as the adequate surgical view to achieve the airway reconstruction. Herein, we describe a successful case of non-intubated anesthesia, performed with a cross-field bronchial suction catheter delivering high-flow oxygen during uniportal VATS sleeve lobectomy in a patient with 
severe chronic obstructive pulmonary disease (Video 1). We present the following case in accordance with the CARE reporting checklist (available at https://jovs.amegroups. com/article/view/10.21037/jovs-20-158/rc).

\section{Case presentation}

A 71-year-old male (165 cm in height, $46 \mathrm{~kg}$ in weight) was referred to our service complaining two weeks of cough. The chest computed tomography scan showed stenosis and thickened wall of the right upper lobe bronchus (Figure 1A). Bronchoscopy confirmed the stenosis that was caused by endobronchial neoplastic tissue (Figure 1B). The pathologist disclosed squamous carcinoma on the biopsy sample. Tumor staging and functional assessment were performed. To preserve the pulmonary function, right upper sleeve

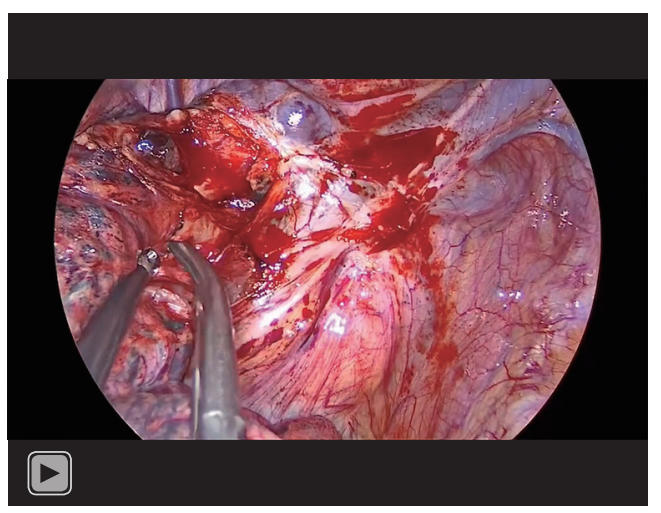

Video 1 Non-intubated uniportal VATS right upper sleeve lobectomy in a patient with compromised lung function. lobectomy under uniportal VATS was planned.

The patient revealed a more than 30 years' smoking history and preoperative pulmonary function testing demonstrated a severe obstructive pulmonary dysfunction (COPD) with an FEV1 of $0.76 \mathrm{~L}$ (35.2\%) and FEV1/FVC ratio of 55.9. The COPD was evaluated and maintained stable before surgery. Oxygen saturation, $\mathrm{PaO}_{2}$ and $\mathrm{PaCO}_{2}$ were within the normal ranges breathing room air. Light to moderate physical activity was well tolerated without shortness of breath. The patient had no other comorbidities and was classified in the level 3 physical status according to the American Society of Anesthesiologists classification (4). Considering the functional assessment and the indication to a complex reconstructive pulmonary resection, a uniportal VATS without tracheal intubation and spontaneous breathing was planned in order to decrease the risk for pulmonary complications possibly occurring after prolonged mechanical ventilation.

Standard monitoring including arterial blood pressure was performed. Intravenous dexmedetomidine of $1 \mu \mathrm{g} / \mathrm{kg}$ (predicted body weight) was infused for 15 minutes with constant speed through an infusion pump. $60 \%$ oxygen was delivered via a facial mask under spontaneous ventilation. The dosage of dexmedetomidine was reduced to $0.5 \mu \mathrm{g} / \mathrm{kg} / \mathrm{h}$. Propofol and remifentanil were infused at the rate of 0.3 and $0.05-0.01 \mathrm{mg} / \mathrm{kg} / \mathrm{h}$ respectively. The facial mask was fixed with a silicone strap and was connected to the ventilator, so, respiratory parameters including tidal volume, respiratory rate and end-tidal $\mathrm{CO}_{2}$ partial pressure could be monitored. The patient was moved to a lateral decubitus position.
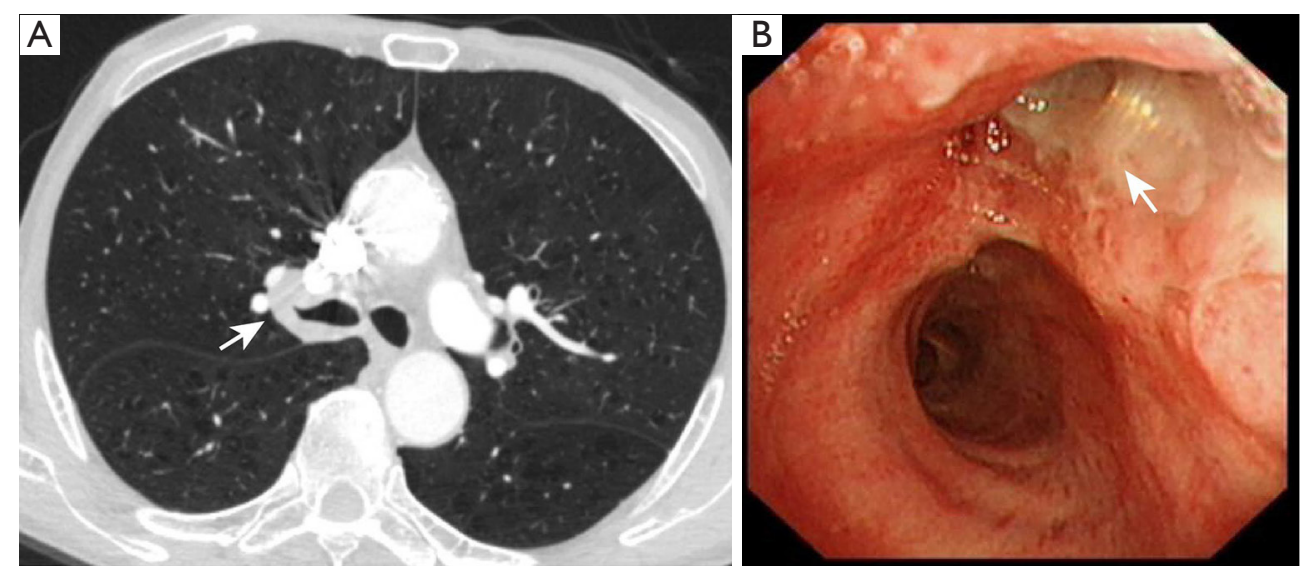

Figure 1 Preoperative imaging features. (A) Computed tomography shows bronchiostenosis and thickened wall in the right upper lobe. (B) Bronchoscopic view of the secondary carina in the right main bronchus. The neoplasm was pointed out with white arrows. 
Intravenous $5 \mu \mathrm{g}$ sufentanil and local $5 \mathrm{~mL}$ of $2 \%$ lidocaine were administered to achieve an adequate level of analgesia before skin incision. The chest was entered through the fourth intercostal space. Pulmonary ligament and the entire right hilum were mobilized with combination of sharp and blunt dissection firstly. The vascular elements of the lobe were transected with staplers. After the right main bronchus and bronchus intermedius were adequately exposed, the intermediate bronchus and right main bronchus were cut circumferentially. Intraoperative histopathology was performed to confirm tumor free margins of the bronchial sleeve. Once the airway was opened, a cross-field bronchial suction catheter from a double lumen tube set delivering $5 \mathrm{~L} / \mathrm{min}$ oxygen was inserted into the right main bronchus stump with thoracoscopic forceps (Figure 2). Circumferential endto-end anastomosis of bronchus intermedius and right

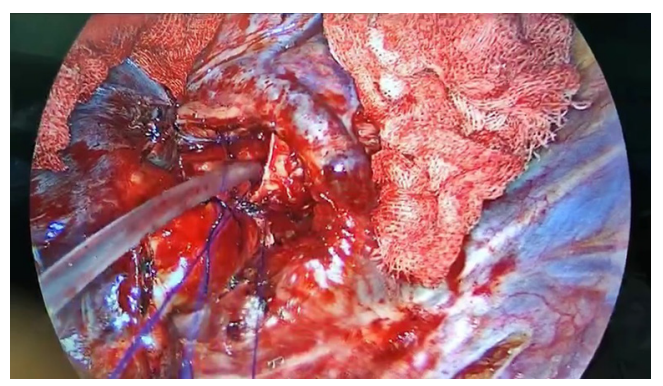

Figure 2 Anastomosis of intermedius to the right main bronchus under the thoracoscopic view. A cross-field high-flow oxygen catheter was inserted to the right main bronchus stump to provide oxygenation to the contralateral lung. main bronchus was performed starting from the pars membranacea to the anterior wall with 3-0 prolene running suture. The small diameter of the bronchial suction catheter allowed an unimpaired view and an adequate operating space. The catheter was removed before tightening the suture. The anastomosis was checked under water with a manually controlled inflation at a pressure of $20 \mathrm{cmH}_{2} \mathrm{O}$ to rule out possible air leak. Manual recruitment maneuvers were performed before chest closure.

The operation was successfully accomplished with a total operative time of 120 minutes. Right lung collapse was well achieved using this technique. The saturation was maintained at more than $95 \%$ throughout the procedure despite a transient duration of tolerable hypercapnia, with highest $\mathrm{paCO}_{2}$ of $66.7 \mathrm{mmHg}$, which was resolved after the anastomosis (Table 1). The patient did not complain any pain or any discomfort and was satisfied with the anesthesia. No pulmonary complications were reported during the hospital stay and the patient was discharged on the postoperative day 5 .

All procedures performed in this study were in accordance with the ethical standards of the institutional and national research committees and with the Helsinki Declaration (as revised in 2013). Written informed consent was obtained from the patient.

\section{Discussion}

Both COPD and general anesthesia are independent risk factors for postoperative pulmonary complications due to the numerous effects on the respiratory system (1). Non-

Table 1 Arterial blood gas analysis before, during and after the operation

\begin{tabular}{|c|c|c|c|c|c|}
\hline Items & $\mathrm{FiO}_{2}$ & $\mathrm{pH}$ & $\mathrm{PaO}_{2}(\mathrm{mmHg})$ & $\mathrm{PaCO}_{2}(\mathrm{mmHg})$ & $\mathrm{SaO}_{2}(\%)$ \\
\hline T1 & 0.6 & 7.420 & 289.2 & 36.2 & 100 \\
\hline T2 & 0.6 & 7.381 & 127.6 & 48.3 & 100 \\
\hline T3 & 0.6 & 7.281 & 100.3 & 66.7 & 96 \\
\hline T5 & 0.6 & 7.40 & 198 & 44.4 & 100 \\
\hline T6 & 0.33 & 7.415 & 133.3 & 32.9 & 100 \\
\hline
\end{tabular}

T0, preoperative arterial blood gas analysis baseline breathing room air; T1, the moment before surgical pneumothorax creation; T2, 30 min after surgical pneumothorax creation (the right main bronchus was intact at the moment); T3, right after the bronchus transection; T4, $1 \mathrm{~min}$ after the anastomosis completion; T5, $5 \mathrm{~min}$ after surgical closure and patient being moved to the spine position; T6, 30 min after patient's total recovery of consciousness breathing $3 \mathrm{~L} / \mathrm{min} \mathrm{O}_{2}$ via a nasal catheter in the post-anesthesia care unit (PACU). 
intubated VATS is a valuable alternative option for these high-risk patients because it avoids intubation and preserves the lungs from mechanical ventilation related injuries (5). The feasibility and safety of non-intubated anesthesia have been reported either in minor and major procedures for patients with compromised lung function $(6,7)$. However, this strategy is rarely reported in case of complex thoracic surgery which may implicate tracheal/ bronchus anastomosis (3).

Non-intubated uniportal thoracoscopic sleeve lobectomy is challenging both for the thoracic surgeons and anesthetists as it requires adequate surgical manipulation space as well as satisfactory oxygenation. In non-intubated VATS, the lung collapse and one-lung ventilation are achieved just opening the chest. The entity of lung collapse progressively increases and is usually comparable to that achieved during intubated one-lung ventilation (8). When the function of the diaphragm is preserved, there is less interference with the functional residual capacity of the ventilating lung. This condition significantly contributes to the maintenance of a good respiratory function and oxygenation (8).

The predisposition to hypercapnia is a frequent finding in non-intubated anesthesia (6). The major contributing factor to hypercapnia is the re-breathing effect of pendulum respiration, which occurs when the air from the nonventilating lung is inhaled into the ventilating lung during inspiration and, conversely, air from the ventilating lung is exhaled into the non-ventilating lung during expiration since the two lungs are not isolated $(2,8)$. The hypercapnia is mostly well tolerated, even in patients with impaired respiratory function, and improves immediately after surgery (8).

In the herein described bronchoplastic procedure, the dissection of the right main bronchus intrinsically resolved the problem of pendulum respiration, which may explain the decline of the $\mathrm{CO}_{2}$ partial pressure from the arterial gas analysis after the anastomosis completion. In this regard, the innovative use of the high-flow oxygen catheter to the proximal stump of the right main bronchus has been helpful for patient oxygenation. The improvement in arterial $\mathrm{O}_{2}$ partial pressure could be attributed to the improved oxygen delivery. Attention should be paid not to use electrocautery during this period to prevent airway fire.

This is the first case to describe the non-intubated anesthesia for right upper sleeve lobectomy under uniportal VATS in a patient with compromised pulmonary function. Neither thoracic epidural anesthesia nor vagal nerve block was applied as the intravenous opioids and local anesthetic were enough to maintain analgesia and suppress cough reflex. To guarantee the safety of the procedure, a double lumen tube was prepared in advance for emergency intubation. In that case, conversion to general anesthesia is mandatory. The thoracic surgeons were proficient in uniportal VATS and the anesthesiologists were trained in performing double lumen tube intubation in the lateral position. The patients should be selected carefully to exclude difficult intubation conditions before deciding to perform non-intubated VATS.

In conclusion, our case suggests that the non-intubated anesthesia is feasible and safe for VATS sleeve lobectomy in patients with reduced pulmonary function. A crossfield oxygen administration may improve oxygenation during the bronchoplastic procedure. As very few reports have been published on this technique, many aspects of this procedure remain undisclosed and disputed. Much more research and technical development is necessary to translate these findings into routine, evidence-based, clinical practice.

\section{Acknowledgments}

Funding: This work was supported by Shanghai Municipal Health Commission Special Clinical Research Foundation (Grant No. 201940366).

\section{Footnote}

Reporting Checklist: The authors have completed the CARE reporting checklist. Available at https://jovs.amegroups. com/article/view/10.21037/jovs-20-158/rc

Peer Review File: Available at https://jovs.amegroups.com/ article/view/10.21037/jovs-20-158/prf

Conflicts of Interest: All authors have completed the ICMJE uniform disclosure form (available at https://jovs. amegroups.com/article/view/10.21037/jovs-20-158/coif). $\mathrm{JL}$ reports that he receives a grant from Shanghai Municipal Health Commission Special Clinical Research Foundation (Grant No. 201940366). The other authors have no conflicts of interest to declare.

Ethical Statement: The authors are accountable for all aspects of the work in ensuring that questions related to the accuracy or integrity of any part of the work are 
appropriately investigated and resolved. All procedures performed in this study were in accordance with the ethical standards of the institutional and national research committees and with the Helsinki Declaration (as revised in 2013). Written informed consent was obtained from the patient.

Open Access Statement: This is an Open Access article distributed in accordance with the Creative Commons Attribution-NonCommercial-NoDerivs 4.0 International License (CC BY-NC-ND 4.0), which permits the noncommercial replication and distribution of the article with the strict proviso that no changes or edits are made and the original work is properly cited (including links to both the formal publication through the relevant DOI and the license). See: https://creativecommons.org/licenses/by-nc-nd/4.0/.

\section{References}

1. Lederman D, Easwar J, Feldman J, et al. Anesthetic considerations for lung resection: preoperative assessment,

doi: 10.21037/jovs-20-158

Cite this article as: Dai L, Chen Z, Vannucci J, Cai J, Lv X, Liu J, Jiang L. Non-intubated uniportal video-assisted thoracoscopy for sleeve lobectomy in a patient with severe chronic obstructive pulmonary disease: a case report. J Vis Surg 2021;7:50. intraoperative challenges and postoperative analgesia. Ann Transl Med 2019;7:356.

2. Gelzinis TA, Sullivan EA. Non-Intubated General Anesthesia for Video-Assisted Thoracoscopic Surgery. J Cardiothorac Vasc Anesth 2017;31:407-8.

3. Bedetti B, Patrini D, Bertolaccini L, et al. Uniportal nonintubated thoracic surgery. J Vis Surg 2018;4:18.

4. Doyle DJ, Goyal A, Bansal P, et al. American Society of Anesthesiologists Classification (ASA Class). StatPearls. Treasure Island FL: (c) 2020, StatPearls Publishing LLC.; 2020.

5. Zhao ZR, Lau RWH, Ng CSH. Anaesthesiology for uniportal VATS: double lumen, single lumen and tubeless. J Vis Surg 2017;3:108.

6. Pompeo E. Pathophysiology of Surgical Pneumothorax in the Awake Patient. Awake Thoracic Surgery 2012:9-18. doi: 10.2174/978160805288211201010009.

7. Okuda K, Nakanishi R. The non-intubated anesthesia for airway surgery. J Thorac Dis 2016;8:3414-9.

8. Pompeo E. State of the art and perspectives in nonintubated thoracic surgery. Ann Transl Med 2014;2:106. 